\title{
Supporting Information: Armoring the interface with surfactant to prevent the adsorption of monoclonal antibody
}

\author{
Ankit D. Kanthe, ${ }^{\dagger}$ Mary Krause,,${ }^{\ddagger}$ Songyan Zheng, ${ }^{\ddagger}$ Andrew llott,,$\ddagger$ Jinjiang

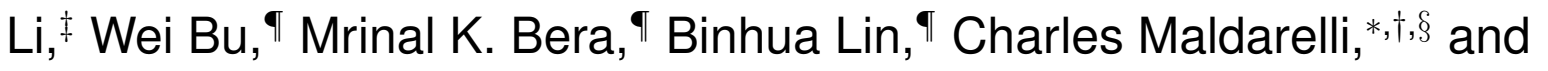 \\ Raymond S. Tu*, \\ $\dagger$ Department of Chemical Engineering, The City College of New York, NY 10031 \\ $\ddagger$ Drug Product Science and Technology, Bristol-Myers Squibb, New Brunswick, NJ, 08901 \\ \ChemMatCARS, Center for Advanced Radiation Sources, University of Chicago, Chicago, IL \\ 60637 \\ $\S$ Levich Institute, The City College of New York, NY 10031 \\ E-mail: cmaldarelli@ccny.cuny.edu; tu@ccny.cuny.edu
}

\section{Langmuir Adsorption Fit}

The dynamic surface tension of mAbs with and without PS80 was studied using the pendant bubble tensiometry. The dynamic surface tension of the excipient alone in $20 \mathrm{mM}$ histidine buffer $\mathrm{pH} 5.8$ was recorded for the concentration range of $0.01-2.00 \times \mathrm{CMC}\left(1.2 \times 10^{-4}-2.4 \times 10^{-2} \mathrm{mM}\right)$. The data was recorded from one second up to three hours. Figure $1 \mathrm{~b}$ shows the representative dynamic surface tension of PS80 at ten different concentrations. PS80 showed a decrease in the surface tension value as the concentration was increased. For lower concentrations of PS80 $(<0.05 \times$ CMC $\left.\left(6.0 \times 10^{-4} \mathrm{mM}\right)\right)$, a continuous decrease in the surface tension value was observed and no 
equilibrium in the surface tension value was achieved even after a long time period (10000 s). On the other hand, for concentrations between $0.05-0.30 \times \mathrm{CMC}\left(6.0 \times 10^{-4} \mathrm{mM}\right.$ and $3.6 \times 10^{-3}$ $\mathrm{mM}$ ), there was a rapid decrease in the surface tension value initially for about $5000 \mathrm{~s}$ due to the availability of monomers at high concentrations of PS80. The decrease in the surface tension value then slows down after the initial time period ( $5000 \mathrm{~s}$ ) and the change in the surface tension value observed was only $\pm 2 \mathrm{mN} / \mathrm{m}$. For concentrations 0.50 and $0.75 \times \mathrm{CMC}(0.006 \mathrm{mM}$ and 0.009 $\mathrm{mM}$ ), the surface tension initially decreases at a faster rate (faster dynamics at early time scales) for about $3500 \mathrm{~s}$, slowing down further with a change of only $\pm 1 \mathrm{mN} / \mathrm{m}$ in the surface tension value. This indicated that equilibrium surface tension was achieved. Furthermore, for concentration $>$ CMC $(0.012 \mathrm{mM})$, the change in the surface tension value was only $\pm 0.2 \mathrm{mN} / \mathrm{m}$ after $2200 \mathrm{~s}$. The critical micelle concentration (CMC) for PS80 was obtained from the equilibrium surface tension vs surface concentration plot. The equilibrium surface tension was obtained from the long-time asymptotes. Based on this, the CMC for PS80 was found to be around $0.012 \mathrm{mM}$.

Table S1: The molecular weight (MW), number of electrons (e) and molecular volume $(\mathcal{V})$ for the antibody (mAb-1 and mAb-2), PS80 and water used in the analysis of XR data.

\begin{tabular}{cccc} 
Component & $\begin{array}{c}\text { MW } \\
(\mathrm{g} / \mathrm{mol})\end{array}$ & $\mathrm{e}$ & $\begin{array}{c}\mathcal{V} \\
\left(\AA^{3}\right)\end{array}$ \\
\hline $\mathrm{mAb}-1$ & 145526.44 & 72763 & 133235.5 \\
$\mathrm{mAb}-2$ & 146464.92 & 77840 & 133235.5 \\
$\mathrm{PS} 80$ & 1310 & 716 & 1803 \\
water & 18 & 10 & 30 \\
\hline
\end{tabular}

In order to estimate the maximum surface coverage, $\Gamma_{\infty}$, the equilibrium surface tension $\gamma$ as a function of the bulk concentration $C_{s}$ is fit to the Gibbs equation for the Langmuir adsorption framework, where the adsorption isotherm and equation of state are given by

$$
\begin{gathered}
\Gamma / \Gamma_{\infty}=1 /\left(1+a / C_{s}\right) \\
\gamma=\gamma_{c}+R T \Gamma_{\infty} \ln \left(1-\Gamma / \Gamma_{\infty}\right)
\end{gathered}
$$


where, $\Gamma$ is the surface concentration and a is the ratio of the desorption to adsorption rate constants. Figure S1 shows the surface tension as a function of bulk concentration of PS80 on semilog axis along with the fit using Langmuir parameters (solid line). We note that the fit using Langmuir isotherm is good giving $\Gamma_{\infty}=1.44 \times 10^{-6} \mathrm{~mol} / \mathrm{m}^{2}\left(1.88 \mathrm{mg} / \mathrm{m}^{2}\right)$ and a $=5.09 \times 10^{-9}$ $\mathrm{mol} / \ell$. This can be related to the minimum area per molecule as $A_{\text {molecule }}=1 / \Gamma \mathcal{N}$ giving 115 $\AA^{2} /$ molecule for PS80 at the air-water interface. The dashed line indicates the kink after which the surface tension is constant at higher concentrations giving the CMC of PS80 as $0.012 \mathrm{mM}$.

\section{$\mathrm{X}$-ray reflectivity data analysis}

The data obtained is normalized by the Fresnel Reflectivity $R_{F}\left(Q_{z}\right)$ of the water. $R_{F}\left(Q_{z}\right)$ corresponds to the reflectivity from an ideally smooth surface with a step-function based electron density profile. The normalized reflectivity helps us understand the features of the molecules on the surface (in the form of oscillations) such as the thickness of the sample at the interface. The corresponding electron density profile for the mAb-excipient system is obtained by analyzing the normalized reflectivity curve using first the model-independent and then the slab model based on Parratt formalism. Although the model-independent method produces a good fit to the XR data, the corresponding EDP is not smooth, while the slab model is not an ideal model for the mAb system, the slab-model has been used to successfully model the XR data for the non-slab structures such as nanoparticles. $^{1,2}$ The details of the method are described elsewhere. ${ }^{3,4}$

Briefly, we start the fitting of the reflectivity data by assuming a layer of uniform electron density with an appropriate thickness. As a first initial guess we used the thickness greater than the length of the $\mathrm{mAb}(\approx 180 \AA)$. In order to get the structural information of the adsorbed molecules along the surface normal direction, the layer was then further subdivided into additional sub-layers each being described by thickness, electron density and roughness. The proposed EDP then undergoes an iterative procedure in such a way that the mean square variation, $\left(\chi^{2}\right)$, that is the difference between the calculated and observed XR curves is minimized. During each iteration process, a slab is randomly selected, and its electron density value is changed to a random value between a prede- 
termined value above and below the average electron density of the neighboring slabs. The method was tested to confirm that the same electron density profile was obtained irrespective of a different initial guess for electron density. This in return ensures the convergence of the fitting procedure to a real electron density profile that is unique. The EDP from the model-independent method is then used as the initial guess for the slab-model to get a smooth EDP. As an example the comparison for the XR fit and the EDP for mAb-1 at $0.5 \mathrm{mg} / \mathrm{m} \ell$ using both the methods is shown in Figure S6.

The molecular weight, molecular volume and number of electrons for the studied mAb and excipient is reported in Table S1. The excipient molecular volume was calculated based on its head and tail group volume. The volume of the single hydrocarbon tail in PS80 is estimated to be half the molecular volume of DOPC tail group (984 $\AA^{3}, 492 \AA^{3}$ per PS80 tail). ${ }^{5}$ The PS80 head group is formed primarily by the ethylene oxide units. The volume of the ethylene oxide unit $\left(\mathrm{C}_{2} \mathrm{H}_{4} \mathrm{O}\right)$ is estimated to be $63 \AA^{3}{ }^{6}{ }^{6}$ The volume of $\left(\mathrm{C}_{2} \mathrm{H}_{4} O\right)_{6} \mathrm{OH}$ was reported to be 395 $\AA^{3}$. As PS80 has repeating units of $\left(\mathrm{C}_{2} \mathrm{H}_{4} \mathrm{O}\right)_{n} \mathrm{OH}$ and $\left(\mathrm{C}_{2} \mathrm{H}_{4} \mathrm{O}\right)_{m}$ attached to the tail group. For simplicity, we consider $\mathrm{n}$ to be 18 and $\mathrm{m}$ to be 2 , resulting into the total head group volume as 1311 $\AA^{3}$. The total molecular volume of PS 80 is then $1803 \AA^{3}$.

\section{Surface Concentration Calculation for Single Component System (mAb or PS80)}

For each concentration, the surface concentration is computed from a volume balance at the interface, and a total electron density balance:

$$
\begin{aligned}
& d=\frac{\mathcal{N}}{M W_{s}} \Gamma_{s} \mathcal{V}_{s}+\frac{\mathcal{N}}{M W_{w}} \Gamma_{w} \mathcal{V}_{w} \\
& e_{T}=\frac{\mathcal{N}}{M W_{s}} \Gamma_{s} e_{s}+\frac{\mathcal{N}}{M W_{w}} \Gamma_{w} e_{w}
\end{aligned}
$$

where $d$ is an interfacial depth, $e_{T}$ is the number of electrons per unit area, $\mathcal{V}_{s}$ is the molecular volume of the (hydrated) PS80, $\mathcal{V}_{w}$ is the molecular volume of water, $\Gamma_{s}$ and $\Gamma_{w}$ are the surface mass densities of the surfactant and water, $e_{w}$ and $e_{s}$ are the number of electrons per molecule 
of water and surfactant, $M W_{s}$ and $M W_{w}$ are the molecular weights of PS80 and water and $\mathcal{N}$ is Avogadro's number. The values for the molecular volumes and electrons per molecule are given in the Supporting Information, Table S1.The values of $d$ and $e_{T}$ are obtained from the EDP profile (Figure 4c for PS80 and 4d for antibody of the main text). $d$ is set to a depth for which the density reaches the plateau value of the density of pure water, and $e_{T}$ is the corresponding integrated area under the EDP curves up to that depth. From the above two equations, $\Gamma_{s}$ and $\Gamma_{w}$ are calculated for that set value of $d$ and the process is repeated for a larger value of $d$ to insure that the calculated $\Gamma_{w}$ and $\Gamma_{s}$ do not change. The above equations are also used to calculate the adsorbed amounts of $\mathrm{mAb} \Gamma_{p}$, with the molecular volume and number of electrons in the above equation replaced by the antibody values (Table S1).

\section{Surface Concentration Calculation for Mixed Component System (mAb + PS80)}

In order to quantitatively predict the surface concentration of the surfactant and antibody during co-adsorption, we assume that the surface structure of each component is the same as in their pure (single-component) system for a given bulk concentration. By assuming this, the ratio of water to protein molecules in the mixed system is the same as in the pure protein system $c_{p}=\frac{\Gamma_{w}^{*} M W_{p}}{\Gamma_{p}^{*} M W_{w}}$, and the ratio of water to surfactant molecules is the same in the mixed and the pure surfactant system $c_{s}=\frac{\Gamma_{w}^{*} M W_{s}}{\Gamma_{s}^{*} M W_{w}}$. In these equations, the asterisk represents the concentrations in layers derived from solutions with only surfactant or protein. This assumption can be justified because of the fact that the number of water molecules per unit area was independent of the component (mAb or PS80) and its concentration in each of the pure systems. Thus the surface concentrations can be found from:

$$
e_{T}=a \frac{\mathcal{N}}{M W_{p}} \Gamma_{p}^{*} e_{p}+a \mathcal{N} \frac{M W_{w}}{M W_{p}} c_{p} \Gamma_{p}^{*} e_{w}+(1-a) \frac{\mathcal{N}}{M W_{s}} \Gamma_{s}^{*} e_{s}+(1-a) \mathcal{N} \frac{M W_{w}}{M W_{s}} c_{s} \Gamma_{s}^{*} e_{w}
$$


where $a$, the only unknown to be solved for, is the area percentage of the protein coverage and the surface concentrations of the protein and surfactant are given by $\Gamma_{p}=a \Gamma_{p}^{*}$ and $\Gamma_{s}=(1-a) \Gamma_{s}^{*}$. The value of $e_{T}$ is obtained from the EDP profile (Figure 5b for mAb-1 + PS80 and Figure 5d for mAb-2 + PS80 of the main text). Using this methodology, Tables 2 and 3 in the main text gives the surface concentrations of $\mathrm{mAb}$ and excipient for mixed adsorption layers from solutions with a fixed concentration of $\mathrm{mAb}(0.5 \mathrm{mg} / \mathrm{m} \ell)$ and increasing concentrations of PS80.

\section{Homology modeling and estimate of antibody size}

The 3D atomic structure of mAb-2 was generated from the antibody sequence using the standard homology modeling protocol ${ }^{7}$ in the MOE software. ${ }^{8}$ Template protein structures with high sequence similarity to each domain and the complementarity-determining region (CDR) loop in the $\mathrm{mAb}$ are sought and scored based on the similarity and the quality of the template structure. The model is built from a combination of the highest scoring templates, with the CDR loop templates grafted onto the $V_{L}$ and $V_{H}$ frameworks. A molecular mechanics energy minimization using the Amber10 force field ${ }^{9,10}$ is then performed on the model to further refine the atomic coordinates and relieve strained geometries. The dimensions of the Fab and Fc domains, as well as the overall extent of the antibody are shown in Figure S4. They were estimated by measuring the relevant inter-atomic distances from the homology model.

\section{Structural Orientation of mAb on surface adsorption}

The possible orientations of the antibody adsorbing to an interface are shown in Figure S5 - (i) flat on (Fab and Fc lying on the surface); (ii) side-on (one Fc and one Fab lying on the surface); (iii) end on - Fab down or head on (both Fabs lying on the surface and (iv) end on - Fab up or tail on (Fc lying on the surface). 


\section{References}

(1) Wang, W.; Lawrence, J. J.; Bu, W.; Zhang, H.; Vaknin, D. Two-Dimensional Crystallization of Poly(N-isopropylacrylamide)-Capped Gold Nanoparticles. Langmuir 2018, 34, 83748378, PMID: 29947524.

(2) You, S. S.; Heffern, C. T.; Dai, Y.; Meron, M.; Henderson, J. M.; Bu, W.; Xie, W.; Lee, K. Y. C.; Lin, B. Liquid Surface X-ray Studies of Gold Nanoparticle-Phospholipid Films at the Air/Water Interface. Journal of Physical Chemistry B 2016, 120, 9132-9141.

(3) Bu, W.; Yu, H.; Luo, G.; Bera, M. K.; Hou, B.; Schuman, A. W.; Lin, B.; Meron, M.; Kuzmenko, I.; Antonio, M. R.; Soderholm, L.; Schlossman, M. L. Observation of A Rare Earth Ion-Extractant Complex Arrested at the Oil-Water Interface During Solvent Extraction. Journal of Physical Chemistry B 2014, 118, 10662-10674.

(4) Pershan, P. S.; Schlossman, M. Liquid Surfaces and Interfaces: Synchrotron X-Ray Methods; Cambridge University Press: Cambridge, 2012.

(5) Wadsäter, M.; Barauskas, J.; Rogers, S.; Skoda, M. W. A.; Thomas, R. K.; Tiberg, F.; Nylander, T. Structural Effects of the Dispersing Agent Polysorbate 80 on Liquid Crystalline Nanoparticles of Soy Phosphatidylcholine and Glycerol Dioleate. Soft Matter 2015, 11, $1140-1150$.

(6) Nagarajan, R. Solubilization of Hydrocarbons and Resulting Aggregate Shape Transitions in Aqueous Solutions of Pluronic $®$ (PEO-PPO-PEO) Block Copolymers. Colloids and Surfaces B: Biointerfaces 1999, 16, 55-72.

(7) Maier, J. K.; Labute, P. Assessment of Fully Automated Antibody Homology Modeling Protocols in Molecular Operating Environment. Proteins: Structure, Function and Bioinformatics 2014, 82, 1599-1610. 
(8) Molecular Operating Environment (MOE), 2016.0801; Chemical Computing Group Inc: Montreal, QC, Canada. 2017; http : / / www . chemcomp. com.

(9) Biller, J. R.; Elajaili, H.; Meyer, V.; Rosen, G. M.; Eaton, S. S.; Eaton, G. R. The Amber Biomolecular Simulation Programs. Journal of MAgnetic Resonance 2013, 236, 47-56.

(10) Case, D.; Darden, T.; Cheatham III, T.; Simmerling, C.; Wang, J.; Duke, R.; Luo, R.; Crowley, M.; Walker, R.; Zhang, W. AMBER 10; University of California: San Francisco, 2008. 2008, 


\section{Supporting Information Figures}

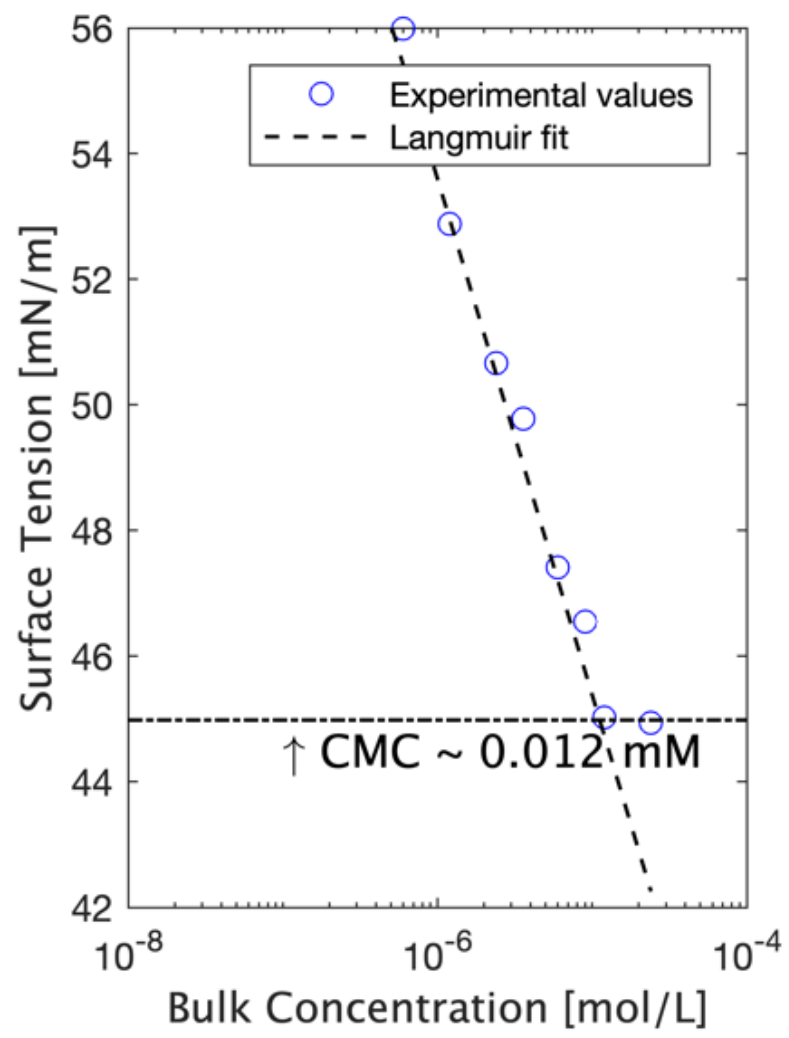

Figure S1: Langmuir adsorption isotherm fit to the equilibrium surface tension data of PS80 obtained from the pendant bubble tensiometry 


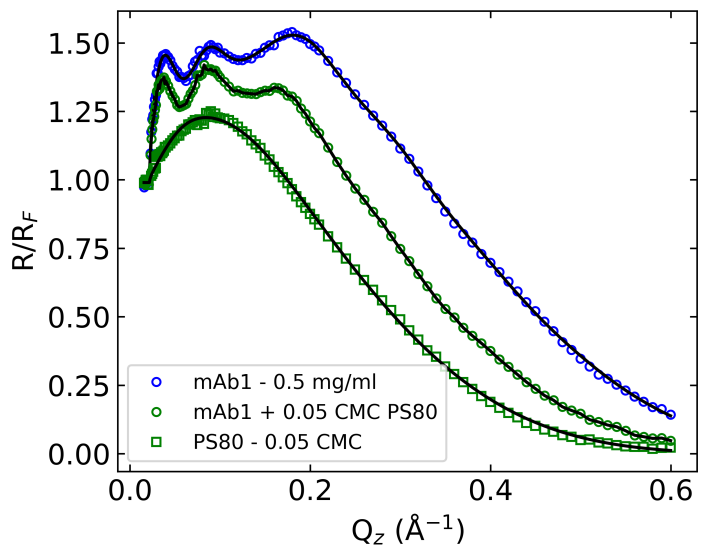

(a)

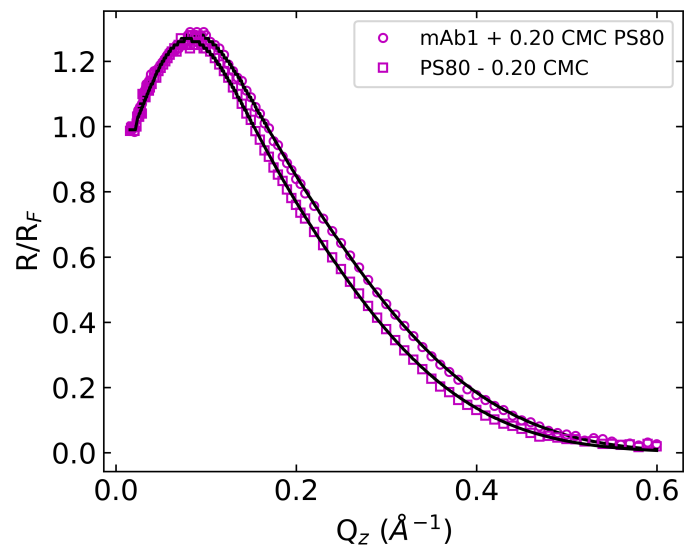

(b)

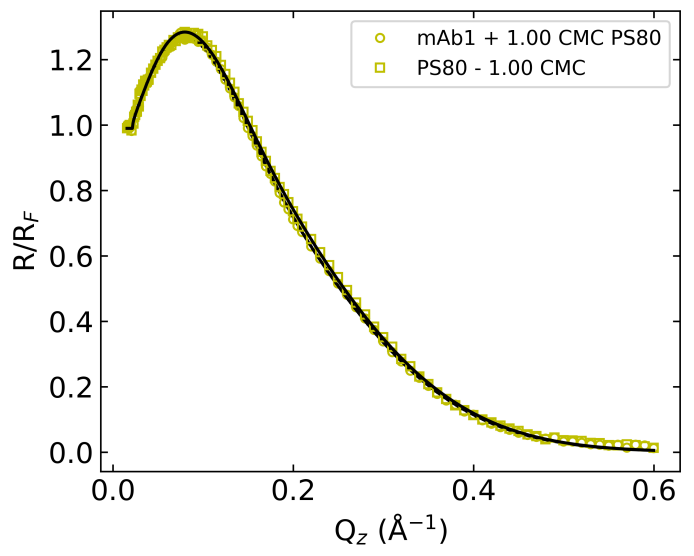

(c)

Figure S2: Overlap of the X-ray reflectivity measurements from adsorbed layers at the air/water interface for the mixed component system (a) X-ray reflectivity measurement of mAb-1 $(0.5 \mathrm{mg} / \mathrm{m} \ell)$ $+0.05 \times$ CMC PS80 concentration (green circle marker) alongside with the pure mAb-1 (0.5 $\mathrm{mg} / \mathrm{m} \ell)$ (blue circle marker) and pure PS80 $(0.05 \times \mathrm{CMC})$ (green square marker) concentration (b) X-ray reflectivity measurement of mAb-1 $(0.5 \mathrm{mg} / \mathrm{m} \ell)+0.2 \times$ CMC PS80 concentration (magenta circle marker) alongside with the pure PS80 $(0.2 \times \mathrm{CMC})$ (magenta square marker) concentration (c) X-ray reflectivity measurement of mAb-1 $(0.5 \mathrm{mg} / \mathrm{m} \ell)+1.0 \times$ CMC PS80 concentration (yellow circle marker) alongside with the pure PS80 $(1.0 \times \mathrm{CMC})$ (yellow square marker) concentration 


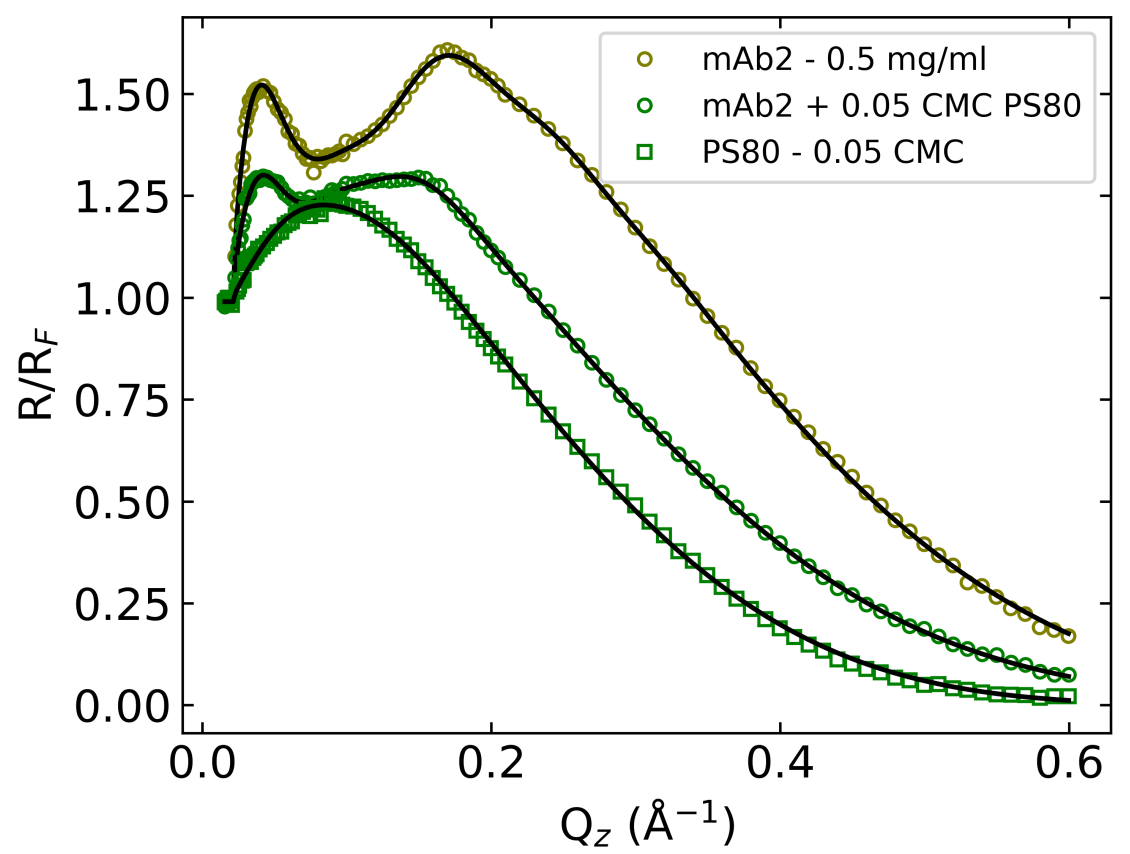

(a)

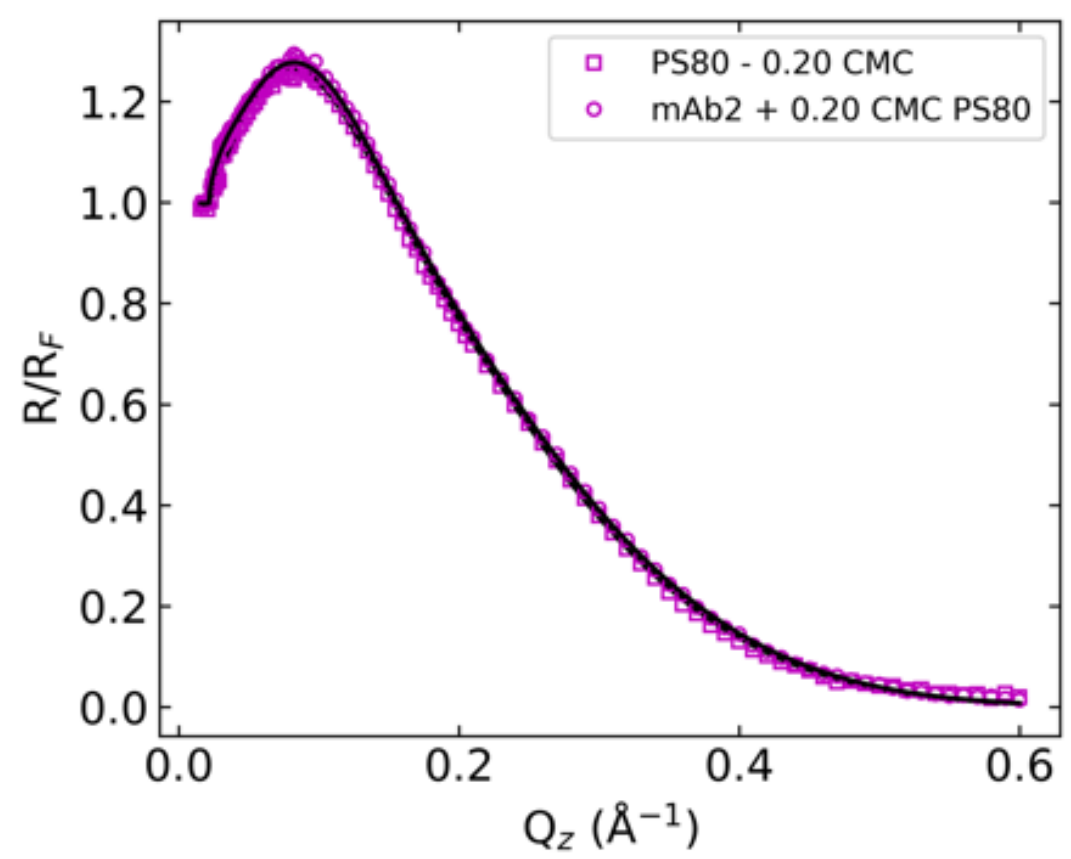

(b)

Figure S3: Overlap of the X-ray reflectivity measurements from adsorbed layers at the air/water interface for the mixed component system (a) X-ray reflectivity measurement of mAb-2 $(0.5 \mathrm{mg} / \mathrm{m} \ell)$ $+0.05 \times$ CMC PS80 concentration (green circle marker) alongside with the pure mAb-2 $(0.5$ $\mathrm{mg} / \mathrm{m} \ell$ ) (olive circle marker) and pure PS80 $(0.05 \times \mathrm{CMC})$ (green square marker) concentration (b) X-ray reflectivity measurement of mAb-2 $(0.5 \mathrm{mg} / \mathrm{m} \ell)+0.2 \times$ CMC PS80 concentration (magenta circle marker) alongside with the pure PS80 $(0.20 \times \mathrm{CMC})$ (magenta square marker) concentration 


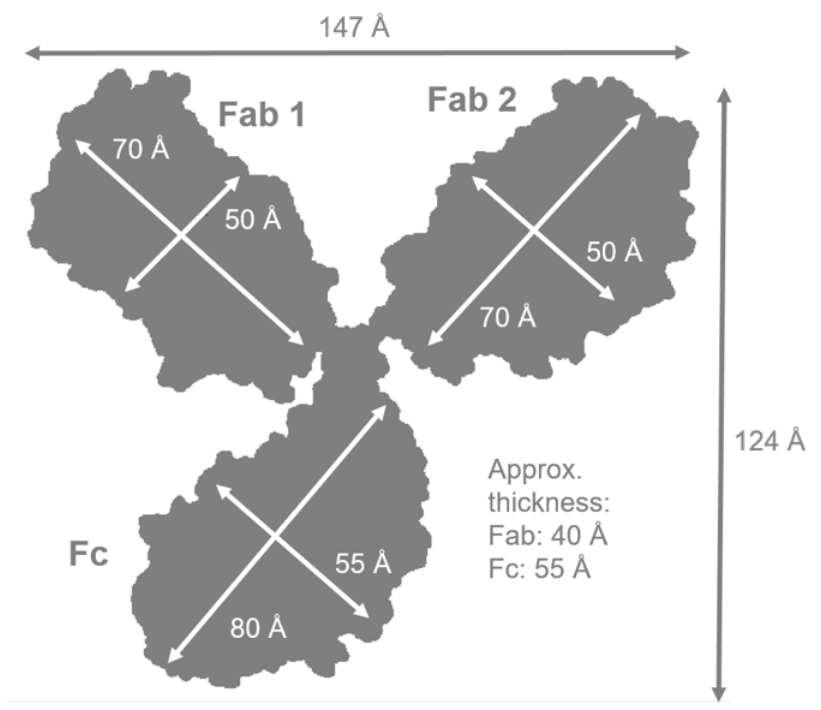

Figure S4: Visualization of the structure and dimensions of a representative IgG molecule

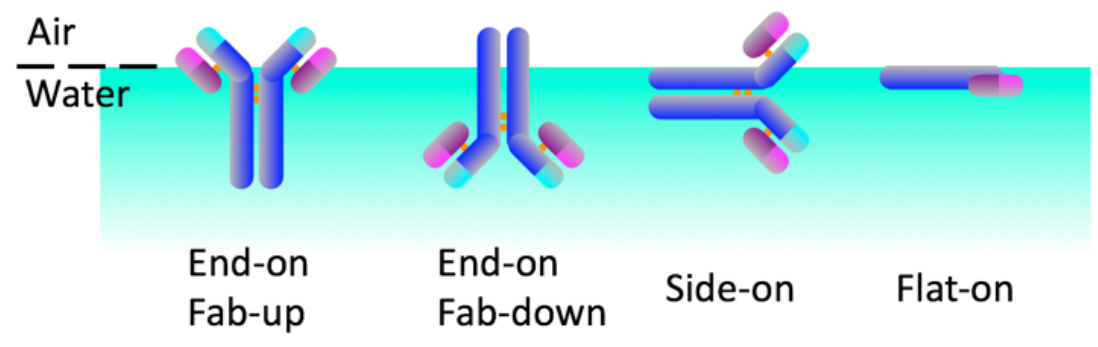

Figure S5: Possible orientation and dimensions of a representative IgG molecule at the air-water interface. The magenta color represents the light chain (variable domains) and the heavy chains are shown in blue. 


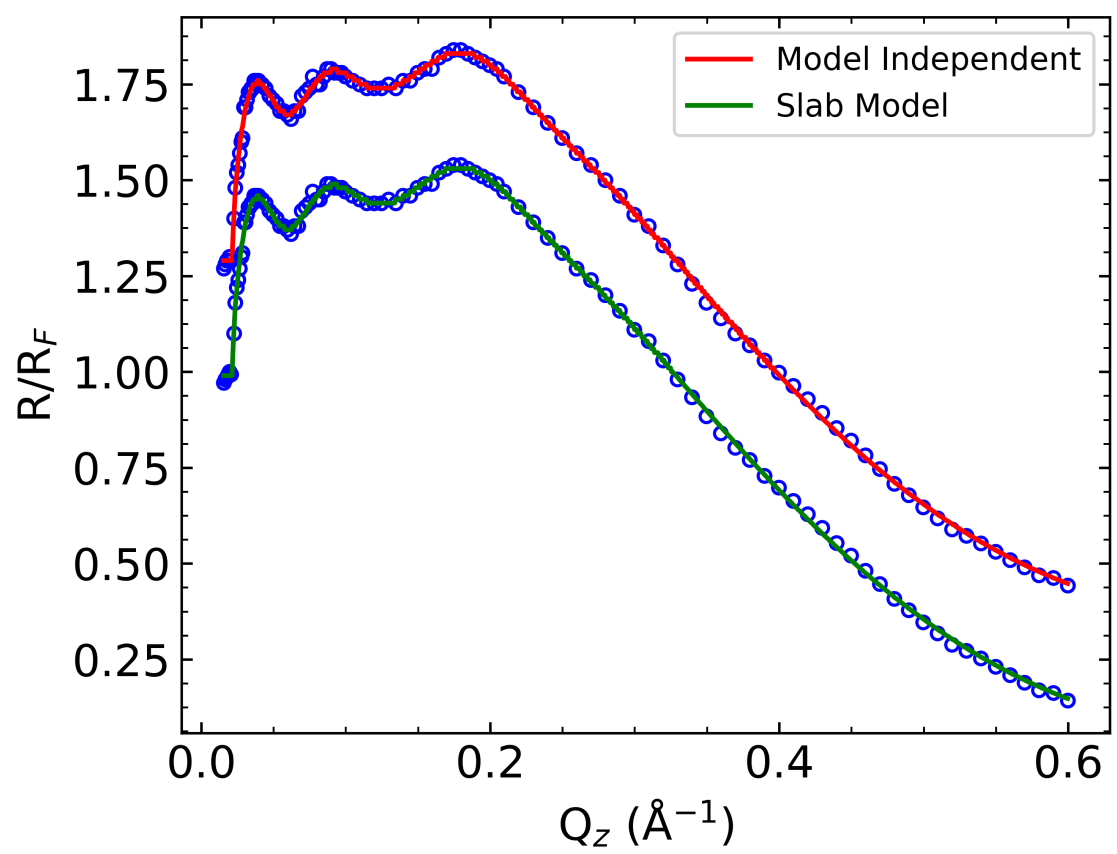

(a)

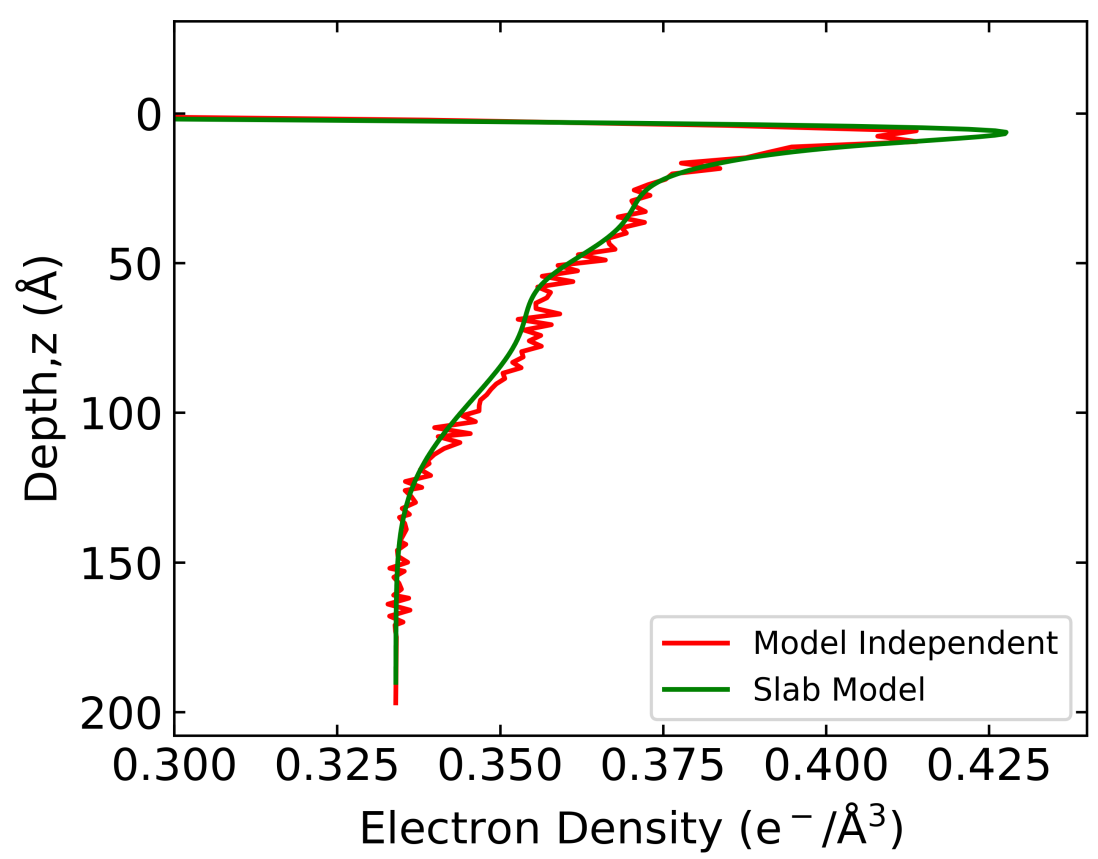

(b)

Figure S6: (a) Normalized X-Ray reflectivity, $R / R_{f}$ (symbols) as a function of the wave function $Q_{z}$ and the Model Independent and Slab Model fits (solid lines) for $\mathrm{mAb}-1$ at $0.5 \mathrm{mg} / \mathrm{m} \ell$. The XR data is shown in blue symbol and the upper XR curve is shifted for clarity, although $R / R_{F} \rightarrow 1$ as $Q_{z} \rightarrow 0$ for all measurements. (b) The corresponding electron density determined by the fits using the Model Independent and the Slab Model. The EDP from the Model Independent method is used as an intitial guess to get a smooth EDP using the Slab Model. 